PROCEEDINGS OF THE

AMERICAN MATHEMATICAL SOCIETY

Volume 138, Number 12, December 2010, Pages 4161-4166

S 0002-9939(2010)10644-5

Article electronically published on August 10, 2010

\title{
ON A CLASS OF FINITARY LIE ALGEBRAS CHARACTERIZED THROUGH DERIVATIONS
}

\author{
MATEJ BREŠAR AND ANTONIO FERNÁNDEZ LÓPEZ
}

(Communicated by Gail R. Letzter)

\begin{abstract}
Let $L$ be an infinite-dimensional simple Lie algebra over a field of characteristic 0 . Then there exist a derivation $d$ on $L$ and $n \geq 2$ such that $d^{n}$ is a nonzero finite rank map if and only if $L$ is finitary and contains a nonzero finite-dimensional abelian inner ideal. This is a partial statement of our main theorem. As auxiliary results needed for the proof we establish some properties of derivations in general nonassociative algebras.
\end{abstract}

\section{INTRODUCTION}

This paper connects two topics that have been studied over the last decade. The first topic concerns finitary Lie algebras (see, e.g., 11) and Lie algebras with minimal inner ideals, a notion introduced by G. Benkart in 4] (see, e.g., 5, 8) . The second topic concerns derivations of algebras such that some of their powers are nonzero finite rank operators (see, e.g., 6, 7]). To the best of our knowledge, so far such derivations were treated only in associative algebras. In this paper we shall examine them in nonassociative algebras, particularly in Lie algebras. The results that we obtain do not give information as detailed as one can get in the associative case where various powerful tools are available; nevertheless, they do give a precise description of the structure of the Lie algebra in question.

Let us be more specific. We will confine ourselves to the consideration of an infinite-dimensional simple Lie algebra $L$ over a field with characteristic 0 . We will show that nonzero finite rank derivations on $L$ do not exist (Theorem 3.1). In our main result we consider the situation where $d$ is a derivation of $L$ such that for some $n \geq 2, d^{n}$ is a nonzero finite rank operator. It turns out that this occurs exactly when $L$ belongs to a certain class of finitary Lie algebras (Theorem 3.2). The proof combines the recently developed structure theory of finitary Lie algebras and Lie algebras with minimal inner ideals with the results on derivations in general nonassociative algebras, obtained in Section 2. The basic result from that section is hidden in the arguments from the recent paper by E. García and M. Gómez Lozano [9].

Received by the editors October 27, 2009.

2010 Mathematics Subject Classification. Primary 17B40, 17B65; Secondary 16W10.

Key words and phrases. Derivation, finite rank, nonassociative algebra, simple Lie algebra, finitary Lie algebra, inner ideal.

The first author was supported by the Slovenian Research Agency (Program No. P1-0288).

The second author was supported by the MEC and Fondos FEDER, MTM2007-61978. 


\section{RESUlts ON GENERAL NONASSOCIATIVE ALGEBRAS}

Let $A$ be a nonassociative algebra. By $\operatorname{End}(A)$ we denote the algebra of all linear maps from $A$ into itself. For every $a \in A$ we define $L_{a}, R_{a} \in \operatorname{End}(A)$ by $L_{a}(x)=a x, R_{a}(x)=x a$. One can define a derivation of $A$ as a map $d \in \operatorname{End}(A)$ satisfying $L_{d(a)}=\left[d, L_{a}\right]$ for every $a \in A$. Equivalently, $R_{d(a)}=\left[d, R_{a}\right]$ for every $a \in A$. Consequently, $L_{d^{m}(a)}=\left[d, L_{d^{m-1}(a)}\right]$ and $R_{d^{m}(a)}=\left[d, R_{d^{m-1}(a)}\right]$ for all $a \in A$ and $m \geq 1$. A simple induction argument shows that this implies that

$$
L_{d^{m}(a)}=\sum_{i=0}^{m}(-1)^{i}\left(\begin{array}{c}
m \\
i
\end{array}\right) d^{m-i} L_{a} d^{i}, \quad R_{d^{m}(a)}=\sum_{i=0}^{m}(-1)^{i}\left(\begin{array}{c}
m \\
i
\end{array}\right) d^{m-i} R_{a} d^{i}
$$

Lemma 2.1. Let $A$ be a nonassociative algebra such that $c A$ is an infinitedimensional space for every nonzero $c \in A$. If $d$ is a derivation of $A$ such that $d^{n}$ has finite rank for some $n \geq 1$, then $d^{2 n-1}=0$.

Proof. From (11) we see that for every $a \in A, L_{d^{2 n-1}(a)}$ lies in the ideal of $\operatorname{End}(A)$ generated by $d^{n}$. Accordingly, $L_{d^{2 n-1}(a)}$ has finite rank; i.e., $d^{2 n-1}(a) A$ is a finitedimensional space. But then $d^{2 n-1}(a)=0$ in view of our assumption.

The next result is deeper.

Theorem 2.2. Let $A$ be a nonassociative algebra over a field $\mathbb{F}$ with char $(\mathbb{F})=0$. If a derivation $d$ of $A$ is such that $d^{n}=0$ for some $n \geq 2$, then $D=d^{n-1}(A)$ satisfies $D^{2}=0$ and $(D A) D \subseteq D$.

An apparently very special case of Theorem 2.2 where $A$ is a Lie algebra and $d$ is an inner derivation, was proved recently by García and Gómez Lozano 9, Theorem 2.3]. Their main intention was to generalize the well-known Kostrikin's lemma [10, p.31] which concerns nilpotent inner derivations on Lie algebras. However, a careful inspection of the proof shows that their arguments can be easily adapted to prove a considerably more general Theorem 2.2. Therefore we shall not give a detailed proof here; let us, however, just point out what the necessary changes are that one has to make.

The first assertion that $D^{2}=0$ is trivial. This follows immediately from $d^{n}\left(a d^{n-2}(b)\right)=0$ by using the Leibniz formula (together with the assumption that $\operatorname{char}(\mathbb{F})=0)$. Proving $(D A) D \subseteq D$ is much more difficult. To demystify this a little bit, let us state explicit formulas for $n=2$ and $n=3$ confirming this inclusion:

$$
\begin{gathered}
d^{2}=0 \Longrightarrow(d(a) b) d(c)=d((d(a) b) c), \\
d^{3}=0 \Longrightarrow\left(d^{2}(a) b\right) d^{2}(c)=d^{2}((d(a) b) d(c)-(d(a) d(b)) c) .
\end{gathered}
$$

Checking the first formula is rather easy, while checking the second one is already not entirely straightforward, as one must also use some other relations derived from $d^{3}=0$. It does not seem completely obvious how to proceed to larger integers $n$. However, an adaptation of the arguments from [9] works. First we notice that (11) implies that

$$
\sum_{i=1}^{n-1}(-1)^{i}\left(\begin{array}{c}
n \\
i
\end{array}\right) d^{n-i} R_{a} d^{i}=0, \sum_{i=1}^{n-1}(-1)^{i}\left(\begin{array}{c}
n \\
i
\end{array}\right) d^{n-i} L_{b} d^{i}=0
$$


for all $a, b \in A$. A glance at the proof of [9, Lemma 2.2] then shows that this lemma holds for $d$ and $R_{a}$ (resp. $L_{b}$ ) playing the role of $X$ and $A$ (here we have referred to the notation in [9]). Next, following the strategy in 9 ] we note that $(D A) D \subseteq D$ will be established by proving that for each pair $a, b \in A$ there exists $\xi \in \operatorname{End}(A)$ such that $R_{d^{n-1}(a)} L_{d^{n-1}(b)}=d^{n-1} \xi$. By (1) we have

$$
\begin{aligned}
(-1)^{n-1} R_{d^{n-1}(a)} L_{d^{n-1}(b)}= & \left(\sum_{i=0}^{n-1}(-1)^{i}\left(\begin{array}{c}
n-1 \\
i
\end{array}\right) d^{n-1-i} R_{a} d^{i}\right) \\
& \times\left(\sum_{i=0}^{n-1}(-1)^{i}\left(\begin{array}{c}
n-1 \\
i
\end{array}\right) d^{i} L_{b} d^{n-1-i}\right) .
\end{aligned}
$$

This is very similar to the beginning of the proof of [9, Theorem 2.3]. The only difference is that in that proof $R_{a}$ and $L_{b}$ are the same operator (which is denoted by $A$, while $X$ is used instead of $d$ ). Fortunately, this does not affect the proof. One just has to follow literally all steps from $[9$ to arrive at the desired conclusion, $R_{d^{n-1}(a)} L_{d^{n-1}(b)}=d^{n-1} \xi$.

Let us also mention that Theorem 2.2 also holds if $\operatorname{char}(\mathbb{F})$ is big enough (depending on $n$ ), not only if it is 0 . See [9].

Corollary 2.3. Let $A$ be a nonassociative algebra over a field $\mathbb{F}$ with $\operatorname{char}(\mathbb{F})=0$. Suppose that $c A$ is an infinite-dimensional space for every nonzero $c \in A$. If there exist a derivation $d$ of $A$ and an integer $n \geq 2$ such that $d^{n}$ is nonzero and has finite rank, then there exists a nonzero finite-dimensional subspace $D$ of $A$ such that $D^{2}=0$ and $(D A) D \subseteq D$.

Proof. Lemma 2.1 shows that $d^{2 n-1}=0$. Let $k$ be such that $d^{k+1}=0$ and $d^{k} \neq 0$. Our assumptions imply that $k \geq n$ and $d^{k}$ has finite rank. Theorem 2.2 therefore tells us that $D=d^{k}(A)$ has the desired properties.

\section{Results on Lie Algebras}

We shall deal with Lie algebras over a field $\mathbb{F}$ with $\operatorname{char}(\mathbb{F})=0$. We recall that a Lie algebra $L$ is said to be nondegenerate if it has no nonzero zero divisors, i.e., if $[x,[x, L]]=0$ implies $x=0$ for any $x \in L$. By [10, Theorem 4.2], the ideal generated by the absolute zero divisors of a Lie algebra over $\mathbb{F}$ is locally nilpotent. Since a simple Lie algebra cannot be locally nilpotent, simple Lie algebras over $\mathbb{F}$ are nondegenerate.

An inner ideal of a Lie algebra $L$ is a subspace $B$ of $L$ such that $[B,[B, L]] \subset B$. An inner ideal $B$ such that $[B, B]=0$ is called an abelian inner ideal. By [4, Theorem 1.12], if $B$ is an abelian minimal inner ideal of a Lie algebra $L$ over $\mathbb{F}$, then $B=[b,[b, L]]$ for any nonzero element $b \in B$.

A Lie algebra is said to be finitary (over $\mathbb{F}$ ) if it is a subalgebra of the Lie algebra $\mathfrak{f g r}(X)$ consisting of all finite rank operators on a vector space $X$ over $\mathbb{F}[\mathbb{1}$.

Theorem 3.1. Let $L$ be a simple Lie algebra over a field $\mathbb{F}$ with $\operatorname{char}(\mathbb{F})=0$. If there exists a nonzero derivation $d$ on $L$ with finite rank, then $L$ is finite dimensional.

Proof. Since $d \neq 0, \operatorname{ad}_{d(a)}=\left[d, \operatorname{ad}_{a}\right]$ is a nonzero inner derivation of finite rank for some $a \in L$. Noticing that the set of all $c \in L$ such that $\operatorname{ad}_{c}$ has finite rank is an ideal of $L$, it follows, by simplicity of $L$, that every inner derivation of $L$ has finite 
rank. Identifying $L$ with $\operatorname{ad}_{L}$ we may therefore assume that $L$ is finitary. Suppose $L$ was not finite dimensional. Then by [1, Theorem 1.1] (rephrased in ring-theoretic terms) we would have that either (a) $L=[A, A]$, where $A$ is an infinite-dimensional simple associative algebra containing an idempotent $e$ such that $e A e$ is a finitedimensional division algebra, that is, $A$ has nonzero socle and can be realized as an algebra of finite rank operators on a left vector space $X$ over the division algebra $\Delta=e A e$ [2, Theorems 4.3.8 and 4.3.9], or (b) $L=[K, K]$ for $K=\operatorname{Skew}(A, *)$, where $A$ is as in (a) and $*$ is an involution.

Suppose first that $L=[A, A]$ is as in (a). Then $X$ is infinite dimensional over $\Delta$; equivalently, $A$ is not Artinian and therefore it contains an infinite sequence $\left\{e_{n}\right\}$ of nonzero pairwise orthogonal idempotents. Then, for $n \neq m, e_{n} A e_{m}=$ $\left[e_{n} A e_{m}, e_{m}\right] \subset[A, A]$. Take a nonzero element $a_{12} \in e_{1} A e_{2}$. Then, for each $n \geq 3$, $\left[a_{12}, e_{2} A e_{n}\right]=a_{12} A e_{n} \neq 0$. Moreover, since the subspaces $e_{1} A e_{n}$ form a direct sum, the inner derivation determined by $a_{12}$ has infinite rank, a contradiction.

Suppose then that $L=[K, K]$ as in (b). As in the previous case, $A$ is not Artinian. Then A contains an infinite sequence $\left\{e_{n}\right\}$ of symmetric pairwise orthogonal nonzero idempotents (of rank one when the involution is of transpose type, and of rank two when it is of symplectic type [2, Proposition 4.6.2]). We claim that for any $a_{n m} \in e_{n} A e_{m}$, with $n \neq m, a_{n m}-a_{n m}^{*} \in[K, K]$. Indeed, let $r$ be a natural number different from $n$ and $m$. By simplicity of $A$, we have $\left(e_{n} A e_{r}\right)\left(e_{r} A e_{m}\right)=e_{n}\left(A e_{r} A\right) e_{m}=e_{n} A e_{m}$. Thus we can write $a_{n m}=\sum_{i=1}^{k} x_{i} y_{i}$, with $x_{i} \in e_{n} A e_{r}, y_{i} \in e_{r} A e_{m}$. Hence

$$
a_{n m}-a_{n m}^{*}=\sum\left(x_{i} y_{i}-y_{i}^{*} x_{i}^{*}\right)=\sum\left[x_{i}-x_{i}^{*}, y_{i}-y_{i}^{*}\right] \in[K, K] .
$$

Now take a nonzero element $a_{12} \in e_{1} A e_{2}$. For any $n \geq 3$, take $b_{2 n} \in e_{2} A e_{n}$ such that $a_{12} b_{2 n}$ is nonzero. We have $\left[a_{12}-a_{12}^{*}, b_{2 n}-b_{2 n}^{*}\right]=a_{12} b_{2 n}-b_{2 n}^{*} a_{12}^{*} \in e_{1} A e_{n} \oplus e_{n} A e_{1}$, which proves that the inner derivation determined by $a_{12}-a_{12}^{*}$ has infinite rank, again a contradiction. Hence $L$ is finite dimensional, as claimed.

It would be interesting to find a different proof of Theorem 3.1, avoiding Baranov's classification [1. Then one might hope to prove this result for more general classes of Lie algebras. Of course, some restrictions on the Lie algebra in question are necessary. After all, just take an infinite-dimensional abelian Lie algebra. A somewhat more sophisticated example, indicating the delicacy of the problem, can be given as follows. Let $M$ be a finite-dimensional simple Lie algebra, and let $L=M \oplus M \oplus M \oplus \cdots$ be a direct sum of countably many copies of $M$. Note that $\operatorname{ad}_{a}$ is nonzero and has finite rank for every nonzero $a \in L$. However, $L$ is infinite-dimensional.

Theorem 3.2. Let $L$ be an infinite-dimensional simple Lie algebra over a field $\mathbb{F}$ with $\operatorname{char}(\mathbb{F})=0$. Then the following conditions are equivalent:

(i) There exists a derivation $d$ on $L$ such that $d^{2}$ is nonzero and has finite rank.

(ii) There exist a derivation $d$ on $L$ and $n \geq 2$ such that $d^{n}$ is nonzero and has finite rank.

(iii) L contains a nonzero finite-dimensional abelian inner ideal.

(iv) $L$ is finitary and there exists a nonzero linear operator $a \in L \subset \mathfrak{F}(X)$ such that $a^{2}=0$.

Moreover, if $\mathbb{F}$ is algebraically closed, then the existence of a nonzero square zero operator in (iv) is automatic. 
Proof. (i) $\Rightarrow$ (ii). Trivial.

(ii) $\Rightarrow$ (iii). Theorem 3.1 implies that $\operatorname{ad}_{c}(A)=[c, A]$ is an infinite-dimensional space for every nonzero $c \in L$. Corollary 2.3 therefore tells us that there exists a nonzero finite-dimensional subspace $D$ of $A$ such that $[D, D]=0$ and $[[D, A], D] \subseteq$ $D$. That is, $D$ is a nonzero finite-dimensional abelian inner ideal.

(iii) $\Rightarrow$ (iv). Since $L$ is simple, nondegenerate and contains a finite-dimensional abelian minimal inner ideal, we have by [8, Theorem 5.1] that either (a) $L$ is a finitedimensional simple Lie algebra over its centroid $\Gamma$, (b) $L=[A, A] / Z(A) \cap[A, A]$, where $A$ is a simple associative algebra containing an idempotent $e$ such that $e A e$ is a finite-dimensional division algebra [8, Proposition 4.7(i)] and $A$ is not a division algebra [3, Corollary 3,15], or (c) $L=[K, K] / Z(A) \cap[K, K]$ for $K=\operatorname{Skew}(A, *)$, where $A$ is as in (b), * is an isotropic involution $\left(a^{*} a=0\right.$ for some nonzero $a \in$ $A$ ), and either $Z(A)=0$ or the dimension of $A$ over $Z(A)$ is greater than 16 , 8 , Proposition 4.14].

Let $B$ be a (finite-dimensional) abelian minimal inner ideal of $L$ and fix a nonzero element $b$ in $B$. Then $b=[b,[b, a]]$ for some $a \in L$ and the mapping $\alpha \mapsto \alpha b=$ $\alpha[b,[b, a]]=[b,[b, \alpha a]]$ defines a linear isomorphism of $\Gamma$ into $B$, so $\Gamma$ is finite dimensional over the base field, and hence the case (a) cannot occur. If $L$ is as in (b), then $A$ is simple with nonzero socle, so it can be regarded as an algebra of finite rank operators on a left vector space $X$ over the division algebra $\Delta=e A e[2$, Theorem 4.3.7]. Since $\Delta$ is finite dimensional, $X$ is infinite dimensional over $\Delta$ and $A$ is not Artinian, so $Z(A)=0$ and $L=[A, A]$ is a finitary special linear algebra [1, Proposition 6.1], which clearly contains a nonzero operator $a$ with $a^{2}=0$. Suppose then that $L$ is as in $(c)$. As above, $Z(A)=0$, so $L=[K, K]$. Now it follows from the structure theorem for simple associative rings with involution and nonzero socle [2. Theorem 4.6.8], and the fact that the division algebra $\Delta$ is finite dimensional, that $L$ is finitary. Using the determination of the inner ideals of $L=[K, K]$ given in [5. Theorem 6.6], we observe that the only possibility for the nonexistence of a nontrivial abelian inner ideal of finite dimension is that $L$ is the finitary orthogonal algebra defined by an infinite-dimensional vector space $X$ with a nondegenerate symmetric bilinear form not containing a totally isotropic subspace of dimension at least 2. But the existence of a 2-dimensional totally isotropic subspace is equivalent to the existence of a skew operator $a$ of $\operatorname{rank} 2$ such that $a^{2}=0$. Notice finally that if the base field is algebraically closed, then $X$ contains totally isotropic subspaces of any finite dimension, so the existence of a zero square operator in (i) is automatic in this case.

(iv) $\Rightarrow$ (i). Take $d=\operatorname{ad}_{a}$. Then $d^{2}(b)=-2 a b a$ for all $b \in L$. Therefore $d^{2}$ is nonzero and has finite rank.

If $d$ is an inner derivation, then (ii) $\Longrightarrow$ (iv) of Theorem 3.2 is slightly reminiscent of Martindale's result [2, Lemma 6.1.2 (ii)], which is of fundamental importance in the theory of generalized polynomial identities of prime rings. One of our intentions when working on this note was to give some indications that the theory of generalized polynomial identities of Lie algebras is plausible.

\section{ACKNOWLEDGEMENT}

The authors would like to thank Esther García for helpful discussions. 


\section{REFERENCES}

[1] A. A. Baranov, Finitary simple Lie algebras, J. Algebra 219 (1999), 299-329. MR 1707673 (2000f:17011)

[2] K. I. Beidar, W. S. Martindale III, and A. V. Mikhalev, Rings with generalized identities. Pure and Applied Mathematics, vol. 196, Marcel Dekker, Inc., New York, Basel, Hong Kong, 1996. MR.1368853 (97g:16035)

[3] G. Benkart, The Lie inner ideal structure of associative rings, J. Algebra 43 (1976), 561-584. MR0435149 (55:8110)

[4] G. Benkart, On inner ideals and ad-nilpotent elements of Lie algebras, Trans. Amer. Math. Soc. 232 (1977), 61-81. MR0466242 (57:6122)

[5] G. Benkart and A. Fernández López, The Lie inner ideal structure of associative rings revisited, Comm. Algebra 37 (2009), 3833-3850. MR2573222

[6] M. Brešar and D. Eremita, The lower socle and finite rank elementary operators, Comm. Algebra 31 (2003), 1485-1497. MR 1971073 (2004b:16020)

[7] M. Brešar, D. Eremita, and T.-L. Wong, On commutators and derivations in rings, J. Algebra 278 (2004), 704-724. MR2071661 (2005d:16056)

[8] C. Draper, A. Fernández López, E. García, and M. Gómez Lozano, The socle of a nondegenerate Lie algebra, J. Algebra 319 (2008), 2372-2394. MR.2388311(2010a:17045)

[9] E. García and M. Gómez Lozano, A note on a result of Kostrikin, Comm. Algebra 37 (2009), 2405-2409. MR 2536928 (2010g:17021)

[10] A.I. Kostrikin, Around Burnside. A Series of Modern Surveys in Mathematics. SpringerVerlag, Berlin, Heidelberg, New York, London, Paris, Tokyo, Hong Kong, 1990. MR.1075416 (91i:20038)

Faculty of Mathematics and Physics, University of Ljubljana, Jadravska ulica 19, SI-1000 Ljubluana, Slovenia - ANd - Faculty of Natural Sciences and Mathematics, University of Maribor, Krekova 2, Si-2000 Maribor, Slovenia

E-mail address: matej.bresar@fmf.uni-lj.si

Departamento de Álgebra, Geometría y Topología, Universidad de Málaga, 29071 Malaga, Spain

E-mail address: emalfer@uma.es 